\title{
Fracture statistics of brittle materials: Weibull or normal distribution
}

\author{
Chunsheng Lu, ${ }^{1,2}$ Robert Danzer, ${ }^{1}$ and Franz Dieter Fischer ${ }^{3}$ \\ ${ }^{1}$ Institut für Struktur- und Funktionskeramik, Montanuniversität Leoben, A-8700 Leoben, Austria \\ ${ }^{2}$ State Key Laboratory of Nonlinear Mechanics, Institute of Mechanics, Academia Sinica, Beijing 100080, China \\ ${ }^{3}$ Institut für Mechanik, Montanuniversität Leoben, A-8700 Leoben, Austria
}

(Received 30 November 2001; revised manuscript received 22 February 2002; published 13 June 2002)

The fit of fracture strength data of brittle materials $\left(\mathrm{Si}_{3} \mathrm{~N}_{4}, \mathrm{SiC}\right.$, and $\left.\mathrm{ZnO}\right)$ to the Weibull and normal distributions is compared in terms of the Akaike information criterion. For $\mathrm{Si}_{3} \mathrm{~N}_{4}$, the Weibull distribution fits the data better than the normal distribution, but for $\mathrm{ZnO}$ the result is just the opposite. In the case of $\mathrm{SiC}$, the difference is not large enough to make a clear distinction between the two distributions. There is not sufficient evidence to show that the Weibull distribution is always preferred to other distributions, and the uncritical use of the Weibull distribution for strength data is questioned.

DOI: 10.1103/PhysRevE.65.067102

PACS number(s): 46.50.+a, 62.20.Mk

Brittle materials, such as ceramics, rock, and concrete, etc., have been widely used in engineering for their excellent resistance to heat, corrosion, and wear. But brittle materials also break easily, and their strength, i.e., the maximum stress they can withstand, varies unpredictably from component to component even if a set of nominally identical specimens are tested under the same conditions. Therefore, the strength of a brittle material is not a well defined quantity and has to be described with respect to fracture statistics [1-3]. Furthermore, the assessment of reliability of brittle materials also requires a probability approach.

As is well known, the Weibull distribution with a flexible two-parameter analytic formula has been found to successfully describe a large body of fracture or fragmentation data, especially for brittle materials, which is therefore suggested to be considered first. As Weibull mentioned in his pioneering papers, however, the Weibull distribution should be considered as an empirical one on an equal footing with other distribution functions [4]. The normal or Gaussian distribution is another widely used function. Other possible candidates, which can be used for probability density function of failure, involve the lognormal, power law, and Type I extreme value distributions, etc. [5,6]. In general, we attempt to identify an appropriate model for the data using the so-called goodness-of-fit tests. However, for small sample sizes, it is difficult to distinguish between the Weibull and normal distributions. In this paper, we will propose a simple quantitative method, which can be used to highlight the difference between the Weibull and normal or other favorite distributions, and find out which model is better.

It is often supposed that a small volume in a brittle material is like a chain of many links, and if any link breaks, then the whole material will fail. Based on this weakest-link principle and an empirical function, the cumulative probability of failure of a brittle material subjected to a load $\sigma$, i.e., the Weibull strength distribution can be represented as $F(\sigma)$ $=1-\exp \left(-\left[\left(\sigma-\sigma_{t h}\right) / \sigma_{0}\right]^{m}\right)$, where $\sigma_{0}$ is a normalized material strength, $\sigma_{t h}$ is the threshold stress (below which no failure will occur), and $m$ is the Weibull modulus [4]. Here, the Weibull modulus is a measure of the degree of strength dispersion, and is also called the shape factor. Then, the probability density function of the three-parameter Weibull distribution $f(\sigma)=d F(\sigma) / d \sigma$ is given by

$$
f(\sigma)=\frac{m}{\sigma_{0}}\left(\frac{\sigma-\sigma_{t h}}{\sigma_{0}}\right)^{m-1} \exp \left[-\left(\frac{\sigma-\sigma_{t h}}{\sigma_{0}}\right)^{m}\right] .
$$

In most applications, $\sigma_{t h}$ is usually taken as zero.

On the other hand, if brittle materials are manufactured and handled without special care, their strengths usually exhibit more or less symmetrical distributions, so the normal distribution could be a natural one to apply to these data [5]. For the normal distribution, its probability density function is

$$
f(\sigma)=\frac{1}{\sqrt{2 \pi} \alpha} \exp \left[-\frac{(\sigma-\bar{\sigma})^{2}}{2 \alpha^{2}}\right],
$$

where $\bar{\sigma}$ and $\alpha$ are the mean and standard deviation, respectively.

In order to find the unknown parameters in a distribution function, the usual way is the linear regression (leastsquares) procedure. However, the best estimate of these parameters is by the maximum likelihood method, which shows the smallest coefficient of variation (the ratio of the standard deviation and mean of a random quantity). The likelihood of a given probability density function is defined as $L=\prod_{i=1}^{N} f\left(\sigma_{i}\right)$, and thus its log-likelihood function is $\ln L$ $=\sum_{i=1}^{N} \ln f\left(\sigma_{i}\right)$, where $N$ is the number of strength experiments. Thus, estimates of these parameters can be found by maximizing the log-likelihood function [5]. For the twoparameter Weibull distribution [let $\sigma_{t h}=0$ in Eq. (1)], the equation for determining $m$ from $N$ measured strengths $\sigma_{i}$ is

$$
\frac{\sum_{i=1}^{N} \sigma_{i}^{m} \ln \sigma_{i}}{\sum_{i=1}^{N} \sigma_{i}^{m}}=\frac{1}{m}+\frac{1}{N} \sum_{i=1}^{N} \ln \sigma_{i},
$$

where $m$ can be obtained by an iterative procedure, and then $\sigma_{0}$ is calculated by 


$$
\sigma_{0}^{m}=\frac{1}{N} \sum_{i=1}^{N} \sigma_{i}^{m} .
$$

Similarly, for the normal distribution, it is relatively easy to get the equations for determining $\bar{\sigma}$ and $\alpha$ from a sample of $N$ measurements $\sigma_{i}$, such as

$$
\bar{\sigma}=\frac{1}{N} \sum_{i=1}^{N} \sigma_{i},
$$

and

$$
\alpha^{2}=\frac{1}{N} \sum_{i=1}^{N}\left(\sigma_{i}-\bar{\sigma}\right)^{2} .
$$

Next, to compare strength data with distribution functions, some measure of the goodness of fit between the functions and data is required. The likelihood ratio statistics appears to be the most promising for use in obtaining confidence bounds [7]. Following very similar considerations, the likelihood approach can be extended to making comparisons between models by the Akaike information criterion (AIC), which starts by linking the likelihood to a distance between the true and estimated distributions, and is defined as

$$
A=-2 \ln \hat{L}+2 k
$$

where $\ln \hat{L}$ is the maximum log-likelihood for a given model, $k$ is the number of parameters to be fitted in the model, and the additional factor 2 is a sop to historical precedents [8]. This represents a rough way of compensating for additional parameters and is a useful heuristic measure of the relative effectiveness of different models [9]. For example, if the three-parameter Weibull distribution with $k=3$ prevails over other distributions, such as the normal or two-parameter Weibull distribution with $k=2$, it must demonstrate a significantly better fit to the strength data, that is, $\Delta A=A_{n}-A_{w 3 p}$ $\geqslant 2[10,11]$, where $\Delta A$ is the difference in AIC values, $A_{n}$ and $A_{w 3 p}$ are calculated using the normal and the threeparameter Weibull distribution functions respectively.

Recently, strength data of three ceramic materials, i.e., silicon nitride $\left(\mathrm{Si}_{3} \mathrm{~N}_{4}\right)$, silicon carbide $(\mathrm{SiC})$, and zinc oxide $(\mathrm{ZnO})$, have been tested. More details about the experiments were discussed in Ref. [12]. The data are often arranged in ascending order and each strength is assigned to a failure probability estimate of $F\left(\sigma_{i}\right)=(i-0.5) / N$, where $i$ is the $i$ th specimen and $N$ is the total number of specimens. Then, the related probability density between $\left(\sigma_{k}, \sigma_{k+1}\right)$ can be calculated by $f\left(\sigma_{k}\right)=1 /\left[N\left(\sigma_{k+1}-\sigma_{k}\right)\right]$, which will be used for the comparison to the fitting distribution functions below.

The results for the three brittle materials are presented in Table I. It is obvious to see that the three-parameter Weibull distribution does not greatly improve the fit although an additional parameter is introduced. We cannot say it is better than the two-parameter Weibull distribution, at least from the AIC values obtained here (in all the three cases, $\Delta A=A_{w 3 p}$ $-A_{w 2 p}>0$, where $A_{w 2 p}$ and $A_{w 3 p}$ are the AIC values calcu-
TABLE I. The AIC values calculated, where $\Delta A=A_{n}$ $-\min \left(A_{w 2 p}, A_{w 3 p}\right)$.

\begin{tabular}{crcccc}
\hline \hline Specimen & \multicolumn{1}{c}{$N$} & $A_{w 2 p}$ & $A_{w 3 p}$ & $A_{n}$ & $\Delta A$ \\
\hline $\mathrm{Si}_{3} \mathrm{~N}_{4}$ & 55 & 635.78 & 637.77 & 642.78 & 7.00 \\
$\mathrm{SiC}$ & 75 & 778.31 & 779.83 & 779.68 & 1.37 \\
$\mathrm{ZnO}$ & 109 & 681.29 & 682.90 & 671.53 & -9.76 \\
\hline \hline
\end{tabular}

lated by the two-parameter and three-parameter Weibull distributions, respectively). For convenience sake, therefore, we shall cite the two-parameter Weibull distribution in the discussion below without special indication.

As shown in Table $\mathrm{I}$, for the $\mathrm{Si}_{3} \mathrm{~N}_{4}$ ceramic, the Weibull distribution fits the data better than the normal distribution, but for the $\mathrm{ZnO}$ ceramic, the behavior is just the opposite [13]. In the case of $\mathrm{SiC}$ ceramic, it seems to tend to the Weibull distribution, but the difference is not large enough for us to make a clear distinction between the two distributions. All these features can be visually observed from the plots of their strength density functions, as illustrated in Figs. 1-3, especially for the $\mathrm{Si}_{3} \mathrm{~N}_{4}$ ceramic (Fig. 1) and the $\mathrm{ZnO}$ ceramic (Fig. 3).

It is of interest to scrutinize the influence of the "outlier" points in both small and large strength sides (see Figs. 1-3) on the fit of data. For the $\mathrm{Si}_{3} \mathrm{~N}_{4}$ ceramic, if the smallest strength was deleted from the fit, $\Delta A=4.71$; and if the smallest two data were removed, $\Delta A=1.90$. Similarly for the SiC ceramic, if the smallest one or two strength data was deleted from the fit, $\Delta A=-0.90$ and -2.65 respectively. In the case of $\mathrm{ZnO}$ ceramic, if the smallest strength was removed from the fit, $\Delta A=-11.94$; otherwise if the largest strength was deleted, $\Delta A=-7.29$. Further if both the smallest and largest strength data were taken out of the fit, $\Delta A=-9.50$. It is obvious to see that the "outlier" data play an important role in the choice of distributions although similar conclusions as discussed above could be obtained. The Weibull distribution is more suitable for the fit of smaller strength data.

The size effect is a direct consequence of the Weibull distribution. In other words, the larger the specimen, the

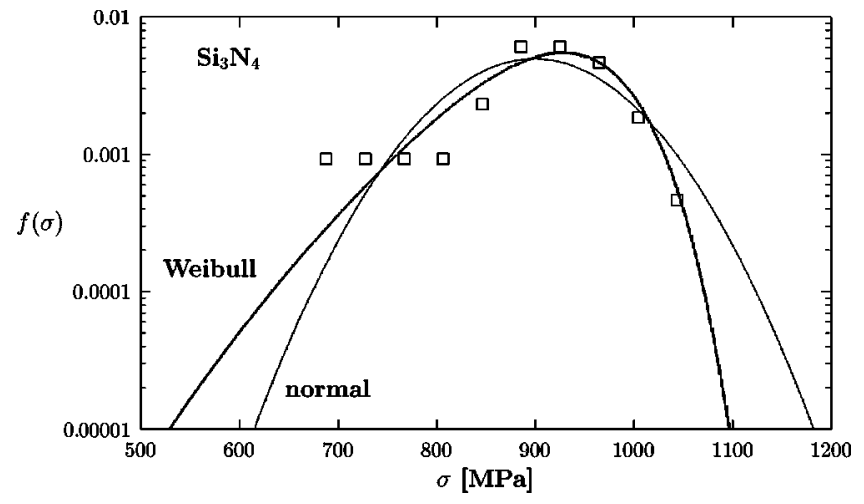

FIG. 1. Strength density functions of the $\mathrm{Si}_{3} \mathrm{~N}_{4}$ ceramic. The fitted parameters to the Weibull distribution are $m=13.89$ and $\sigma_{0}$ $=933.56 \mathrm{MPa}$; and the fitted parameters to the normal distribution are $\bar{\sigma}=899.42 \mathrm{MPa}$ and $\alpha=80.49 \mathrm{MPa}$. 


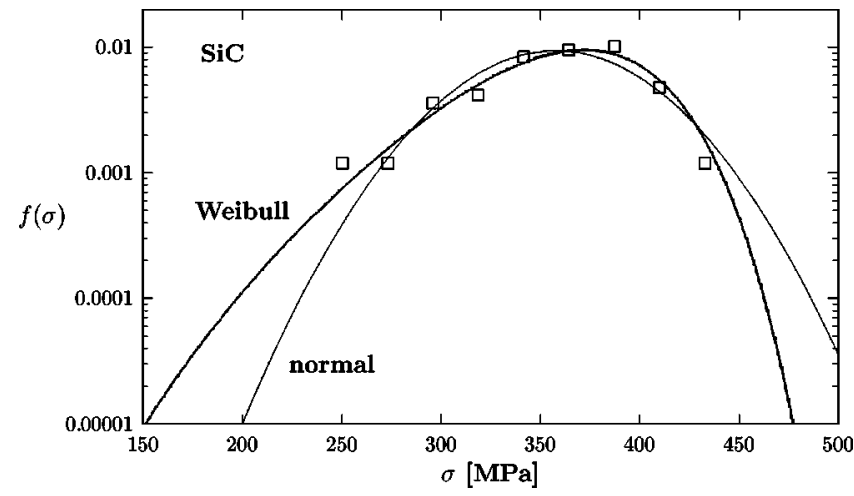

FIG. 2. Strength density functions of the SiC ceramic. The fitted parameters to the Weibull distribution are $m=9.62$ and $\sigma_{0}$ $=376.20 \mathrm{MPa}$; and the fitted parameters to the normal distribution are $\bar{\sigma}=357.87 \mathrm{MPa}$ and $\alpha=42.62 \mathrm{MPa}$.

higher the probability that we could find a large and critical defect and the smaller the mean strength of the corresponding sample $[14,15]$. This size effect can be represented as $V_{1} \sigma_{1}^{m}=V_{2} \sigma_{2}^{m}$ if we suppose two samples of specimens with different sizes $V_{1}$ and $V_{2}$ as well as the same probability of failure. In more general terms, we have $\sigma(\lambda V)$ $\sim \lambda^{-1 / m} \sigma(V)$, where $\lambda$ is the scaling factor. This provides us with another experimental way to check out our results mentioned above. As seen in Fig. 4, further experimental results show the tendency we have discovered [12]. For the $\mathrm{Si}_{3} \mathrm{~N}_{4}$ and $\mathrm{SiC}$ ceramics, the Weibull distribution fits the data better, but in the case of $\mathrm{ZnO}$ ceramic, there is no size effect, that is to say, some characteristic scale may exist and the normal distribution is a possible choice.

In addition, microscopic observations showed that there are very different microstructures in these materials. In the $\mathrm{Si}_{3} \mathrm{~N}_{4}$ and $\mathrm{SiC}$ ceramics, cracklike flaws are sparsely distributed, and thus it is not surprising that their strengths yield the Weibull distribution. But in the $\mathrm{ZnO}$ ceramic, flaws are approximately spherical pores with sharp grooves $[12,16]$. As we know, the $\mathrm{ZnO}$ ceramic is a typical kind of electroceram-

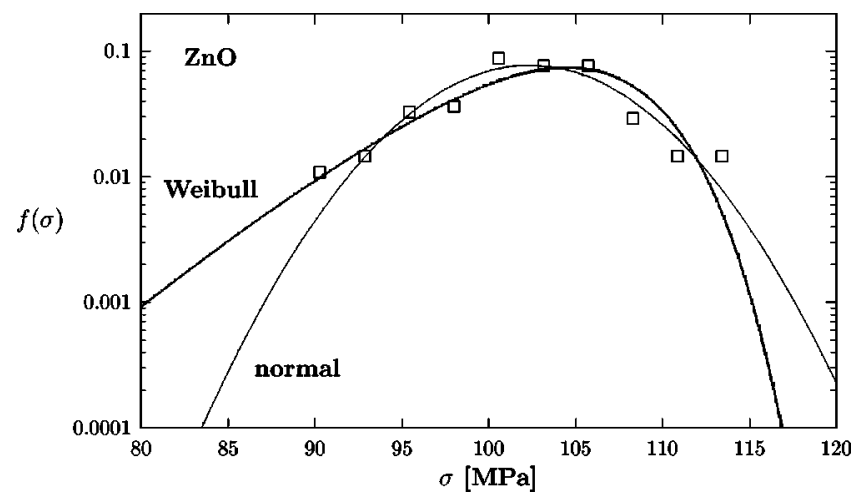

FIG. 3. Strength density functions of the $\mathrm{ZnO}$ ceramic. The fitted parameters to the Weibull distribution are $m=20.92$ and $\sigma_{0}$ $=104.81 \mathrm{MPa}$; and the fitted parameters to the normal distribution are $\bar{\sigma}=102.37 \mathrm{MPa}$ and $\alpha=5.17 \mathrm{MPa}$.

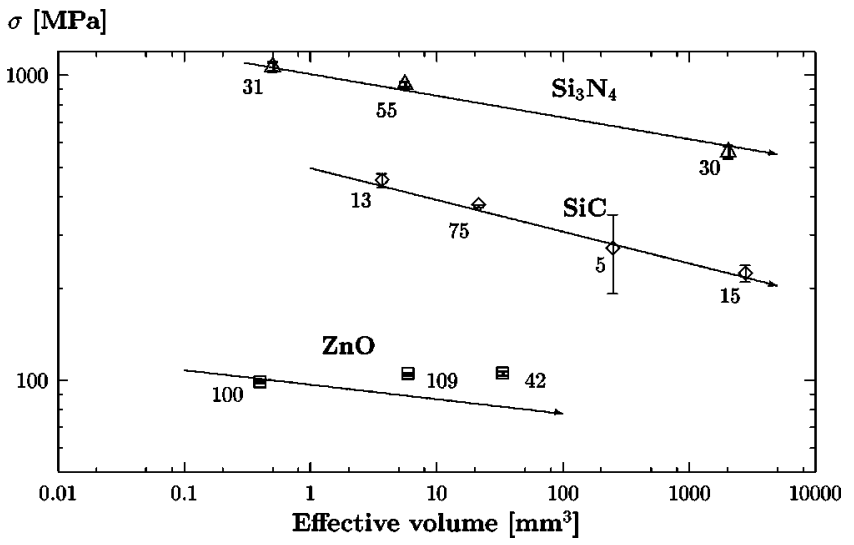

FIG. 4. Experimental results for three ceramics, where numerals are the number of experiments, and error bars refer to $90 \%$ confidence band (the higher the number of tests, the smaller the scatter of data). Solid arrow lines, with the slope of $-1 / m$, indicate the size effect extrapolated by the Weibull distribution.

ics that are applied for varistors and designed with respect to electrical rather than mechanical properties. Thus, they contain a large number of flaws that may act as the origin of fracture. As a consequence of a high porosity (about 5 vol \%) and a pronounced $R$-curve behavior before fracture in the $\mathrm{ZnO}$ ceramic, a group of pores as well as the interaction between them would affect the final fracture rather than only the largest one as the Weibull weakest-link model postulated. Now we attempt to give a simple statistical explanation. Let us suppose that the number of defects in a given sample is $N$, and the failure probability of a defect is $p$. For the sake of simplicity, the interaction between pores is neglected. The failure probability of $n$ defects can thus be written as $P_{N}(n)=\{N ! /[n !(N-n) !]\} p^{n}(1-p)^{N-n}$. As is well known [17], there are two special cases for this binomial distribution in the limit of large $N$. If $p$ is not too small (this seems to be corresponding to the case of $\mathrm{ZnO}$ ceramic), the binomial distribution is approaching the normal distribution. On the contrary, if $p \ll 1$, we have the Poisson distribution $P_{N}(n)$ $=a^{n} \exp (-a) / n$ !, where $a=N p$. Further let $n=0$, one can easily obtain $P_{N}(0)=\exp (-N p)$, and then the weakest-link model can be described in the form $F=1-P_{N}(0)=1$ $-\exp (-N p)$. Based on as few assumptions as possible, a similar formula $F_{S}(\sigma)=1-\exp \left[-\left\langle N_{c, S}(\sigma)\right\rangle\right]$, where $\left\langle N_{c, S}(\sigma)\right\rangle$ indicates the mean number of critical defects in a specimen of size $S$, was proposed [15]. So obviously the Weibull distribution is only a special case of this general distribution function.

Finally it is worth noting that the procedure proposed here can be extended and applied to choose the best distribution among three or more possible distributions, for which AIC as defined in Eq. (7) has the smallest value. It has also been used to investigate the influence of threshold stress to the estimation of the Weibull statistics [18].

In conclusion, we have introduced a simple quantitative procedure to ascertain a better distribution, and further applied it to the fit of strength data of three ceramic materials. 
The results show that there seems to be no sufficient evidence that the Weibull distribution is always in preference tothe normal or other distributions. The uncritical use of the Weibull distribution for strength data is questioned. The careful search for the optimum distribution could however provide the first hint and help us to elucidate the underlying physical mechanisms of fracture.
We gratefully thank Tanja Lube for supplying the strength data and assistance in experiments, and Xaver Remsing for a careful reading of the manuscript. One of the authors $(\mathrm{C} . \mathrm{Lu})$ would like to thank David Vere-Jones for many valuable discussions. This work has been supported by the Lise Meitner Program of the Austrian Science Fund (FWF) under Project No. M587.
[1] B. R. Lawn, Fracture of Brittle Solids, 2nd ed. (Cambridge University Press, Cambridge, 1993).

[2] Statistical Models for the Fracture of Disordered Media, edited by H. J. Herrmann and S. Roux (North-Holland, Amsterdam, 1990).

[3] S. L. Pheonix and R. Raj, Acta Metall. Mater. 40, 2813 (1992); P. L. Leath and P. M. Duxbury, Phys. Rev. B 49, 14905 (1994); W. A. Curtin, Phys. Rev. Lett. 80, 1445 (1998).

[4] W. Weibull, Proc. Ing. Vatenskaps. Akad. 151, 1 (1939); J. Appl. Mech. 18, 293 (1951).

[5] R. H. Doremus, J. Appl. Phys. 54, 193 (1983), and references therein.

[6] E. J. Gumbel, Statistics of Extremes (Columbia University Press, New York, 1958).

[7] D. R. Cox and D. Oakes, Analysis of Survival Data (Chapman \& Hall, London, 1984).

[8] H. Akaike, in Applications of Statistics, edited by P. R. Krishnaiah (North-Holland, Amsterdam, 1977), p. 27; Y. Sakamoto, M. Ishiguro, and G. Kitagawa, Akaike Information Criterion Statistics (Reidel, Dordrecht, 1983).

[9] It is worth noting that the AIC values obtained here should be used with some caution since the amount of experimental data is not very large. For detailed discussion, see D. Daley and D. Vere-Jones, An Introduction to the Theory of Point Processes (Springer, Berlin, 1988).

[10] In typical cases, model differences which would be significant at around the 5\% confidence level correspond to differences in AIC values of around $1.5 \sim 2$.
[11] C. Lu, D. Vere-Jones, and H. Takayasu, Phys. Rev. Lett. 82, 347 (1999); C. Lu and D. Vere-Jones, J. Geophys. Res., [Solid Earth] 106, 11115 (2001).

[12] R. Danzer and T. Lube, in Ceramic Materials and Components for Engines, edited by K. Niihara (Japan Fine Ceramics Association, Tokyo, 1998), p. 683; R. Danzer, in Ceramics: Getting into the 2000's, edited by P. Vicenzini (Techna Srl., Faenza, 1999), p. 379; R. Danzer, T. Lube, and P. Supancic, Z. Metallkd. 92, 773 (2001).

[13] For $\mathrm{ZnO}$, a measured strength $(\sigma=71.5 \mathrm{MPa})$, which is far from the data range $(89 \sim 114.7 \mathrm{MPa})$ in 110 experiments, is believed to be due to other causes and eliminated in the calculation discussed here.

[14] For a review, see Z. P. Bažant, Arch. Appl. Mech. 69, 703 (1999), and references therein; A. Carpinteri, Int. J. Solids Struct. 31, 291 (1994).

[15] R. Danzer, J. Eur. Ceram. Soc. 10, 461 (1992); R. Danzer and T. Lube, in Fracture Mechanics of Ceramics, edited by R. C. Bradt et al., (Plenum Press, New York, 1996), Vol. 11, p. 425.

[16] A. Zimmermann, M. Hoffman, B. D. Flinn, R. K. Bordia, T.-J. Chuang, E. R. Fuller, and J. Rödel, J. Am. Ceram. Soc. 81, 2449 (1998).

[17] L. E. Reichl, A Modern Course in Statistical Physics (University of Texas Press, Texas, 1980).

[18] When experimental data are limited in number and the threshold stress is not too large, numerical simulation has shown that the two-parameter Weibull distribution is still a preferable choice. C. Lu, R. Danzer, and F. D. Fischer J. A. Ceram. Soc. 85, 1640 (2002). 